\title{
Molecular epidemiology and characterization of an outbreak causing Klebsiella pneumoniae clone carrying chromosomally located bla $a_{\mathrm{CTX}-\mathrm{M}-15}$ at a German University-Hospital
}

\author{
Stephen E. Mshana' ${ }^{1}$, Moritz Fritzenwanker ${ }^{2,3}$, Linda Falgenhauer ${ }^{2,3}$, Eugen Domann ${ }^{2,3}$, Torsten Hain²,3,
} Trinad Chakraborty ${ }^{2,3}$ and Can Imirzalioglu ${ }^{2,3^{*}}$

\begin{abstract}
Background: Multi-drug resistant Klebsiella pneumoniae strains are a common cause of health care associated infections worldwide. Clonal spread of Klebsiella pneumoniae isolates carrying plasmid mediated CTX-M-15 have been commonly reported. Limited data is available regarding dissemination of chromosomally encoded CTX-M-15 in Klebsiella pneumoniae worldwide.

Results: We examined 23 non-repetitive ESBL-producing Klebsiella pneumoniae strains isolated from clinical specimens over a period of 4 months in a German University Hospital. All isolates were characterized to determine their genetic relatedness using Pulsed-Field Gel Electrophoresis (PFGE) and Multi Locus Sequence Typing (MLST). PFGE revealed three clusters (B1, B2, and B3) with a sub-cluster (A3) comprising of 10 isolates with an identical PFGE pattern. All strains of the cluster B3 with similar PFGE patterns were typed as ST101, indicating an outbreak situation. The ESBL allele bla $a_{\mathrm{CTX}-\mathrm{M}-15}$ was identified in 16 (69.6\%) of all isolates, including all of the outbreak strains. Within the A3 sub-cluster, the CTX-M-15 allele could not be transferred by conjugation. DNA hybridization studies suggested a chromosomal location of bla CTX-M-15. Whole genome sequencing located CTX-M-15 within a complete ISECP-1 transposition unit inserted into an ORF encoding for a putative membrane protein. PCR-based analysis of the flanking regions demonstrated that insertion into this region is unique and present in all outbreak isolates.
\end{abstract}

Conclusion: This is the first characterization of a chromosomal insertion of bla $a_{\mathrm{CTX}-\mathrm{M}-15}$ in Klebsiella pneumonia ST101, a finding suggesting that in Enterobacteriaceae, chromosomal locations may also act as reservoirs for the spread of bla $a_{\mathrm{CTX}-\mathrm{M}-15}$ encoding transposition units.

Keywords: Hospital, Nosocomial infection, ESBL, Multi-resistance, Klebsiella, Chromosomal insertion

\section{Background}

Klebsiella pneumoniae (K. pneumoniae) is among the most common multi-resistant bacteria causing healthcare associated infections [1-3]. Extended-spectrum $\beta$-lactamase (ESBL) producing $K$. pneumoniae are associated with both hospital and community infections $[1,4]$. Worldwide, there is an increasing number of reports on CTX-M producing

\footnotetext{
* Correspondence: can.imirzalioglu@mikrobio.med.uni-giessen.de

${ }^{2}$ Institute of Medical Microbiology, Justus-Liebig University, , Schubertstrasse 81, 35392 Giessen, Germany

${ }^{3}$ German Center for Infection Research (DZIF), Partner site Giessen-Marburg-Langen, Campus Giessen, Schubertstrasse 81, 35392 Giessen, Germany
}

Full list of author information is available at the end of the article
K. pneumonia isolates, as evidenced from data presented in different multi-centre studies. K. pneumoniae isolates with plasmids harbouring CTX-M-15 have been reported from clinical isolates both from Europe and America [1, 2, 5-7]. The mobility of genetic elements, in particular those conferring antibiotic resistance traits, together with clonal expansion contributes to the persistence of these strains in hospitals and in the community $[4,8]$. There are currently only a few reports of chromosomally encoded CTX-M alleles in Escherichia coli, K. pneumoniae, Salmonella enterica and Proteus mirabilis [8-10]. Recently, K. pneumoniae strains with 
chromosomally integrated CTX-M-15 at an undetermined locus were typed as ST1 in a Spanish study [8].

Here we report the clonal outbreak of ESBL producing $K$. pneumoniae carrying bla $a_{\mathrm{CTX}-\mathrm{M}-15}$ in a distinct chromosomal location.

\section{Methods}

\section{Bacterial isolates}

Twenty-three non-repetitive, phenotypically proven ESBL producing- K. pneumoniae clinical isolates collected consecutively were studied. They represent $11.6 \%$ of all $K$. pneumoniae isolated during the study period. These isolates were taken from miscellaneous specimens including urine, sputum, blood and various swabs over a period of 16 weeks from January 2007 to May 2007. The ESBL phenotype was detected using disk diffusion methods [11]. In addition to routine antimicrobial susceptibility testing by disk diffusion, the Minimal Inhibitory Concentrations (MIC) for cefepime and tigecycline were determined using E-Test stripes (AB BIODISK, Sweden) following the manufacturer's instruction. Isolates with a MIC of $\geq 8 \mathrm{mg} / \mathrm{L}$ for cefepime and a MIC of $\geq 2 \mathrm{mg} / \mathrm{L}$ for tigecycline were considered resistant according to recommendations made by the Clinical Laboratory Standards Institute 2010 (CLSI, USA).

\section{Genetic relatedness and genes encoding for $\beta$-lactamases} All isolates were screened for the presence of CTX-M, TEM and SHV genes using primers and methods described previously [12]. PCR fragments were sequenced with the ABI Prism 3100 sequencer (Life technology/Applied Biosystems, USA). DNA sequence analysis was performed using the DNASTAR software (DNASTAR, USA) and homology searches performed using the NCBI Blast database (http://blast.ncbi.nlm.nih.gov/Blast.cgi).

PFGE was performed according to the Pulse Net protocol of the Centre for Disease Control and Prevention (Atlanta, USA). Strain differentiation by PFGE analysis was achieved by comparison of band patterns using Gelcompar II (Applied Maths, Belgium). Patterns were normalized using the molecular weight marker (PFGE Lambda Marker, New England Biolabs, Germany). The similarity coefficient (SAB) of sample pairs was calculated based on band positions by using the DICE metric $[13,14]$. Dendograms were generated to visualize relationships among the isolates. The cut-off value in the dendograms was calculated at a SAB of 0.97 as a threshold for defining clusters of genetically similar isolates.

Phylogenetic grouping was performed using a rapid method combining gyrA PCR- restricted fragment length polymorphisms analysis (RFLP), parC-PCR and adonitol fermentation as described previously [15]. PCR based replicon typing was done as described by Carattoli et al. [16] to determine the replicons of plasmid in all clinical isolates. To detect sequence type (ST), MLST was performed as described previously [17]. Briefly, PCR for seven housekeeping genes (rpoB, gapA, $m d h, p g i, p h o \mathrm{E}$, $\inf \mathrm{B}$ and $\operatorname{ton} \mathrm{B}$ ) was conducted and the products were directly sequenced and analyzed for single loci variants.

\section{Location of the CTX-M-15 gene}

Conjugation was performed using plate mating experiments as described [18] . Plasmid analysis was done using the method described [19]. The DNA was transferred on a polyvinyl-based membrane using overnight capillary transfer (CUMC Protocol for Southern Blot, New York) and hybridization done using a CTX-M-15 DIG labelled probe (DIG High Prime DNA Labelling and Detection Starter Kit II, Roche, Germany) following manufacturer's instructions.

The whole genome sequence of chromosomal DNA of isolate number 39 was determined. Chromosomal DNA was isolated from an overnight culture with the Purelink DNeasy kit according to the manufacturer's instructions (Invitrogen, Germany). The DNA was then sheared to about $290 \mathrm{bp}$ on the Bioruptor sonication system (Diagenode, Belgium). A chromosomal DNA library was built on the ABI library builder, using the Library Builder $^{\text {Tx }}$ Fragment Core Kit for SOLiD ${ }^{\bullet} 4$ according to the protocol demonstrated by Life Technologies (Life Technologies, Germany). The library was then sequenced on a 316 Ion Torrent Chip using the Ion PGM 200 Sequencing Kit (Life Technologies, Germany). The Ion Torrent PGM (Life Technologies, Germany) produced 2.8 million reads with an average length of $185 \mathrm{bp}$, which amounted to 525.33 Mbp of bases, 324.43 Mbp of which were Q20 or higher quality. The reads were assembled using MIRA 3.4 [20], resulting in 650 contigs larger than or equal to $500 \mathrm{bp}$ with an average coverage of $69.9 \times$. The N50 contig size was $18,876 \mathrm{bp}$. The assembly was improved by manually adding PCR reads in Lasergene Seqman software environment (DNASTAR, USA). A CTX-M-15 carrying insertion

Table 1 Primers used in this study to investigate CTX-M-15 location

\begin{tabular}{ll}
\hline Name & Nucleotide-Sequence $\left(5^{\prime}-3^{\prime}\right)$ \\
\hline DOW2.55 & GCATCCAAGAGATTCTATCG \\
DOW2.5 & CATACACTCCCTTGTACGG \\
MP4b-F & TCCTCGACGTTCACGTCT \\
MP-3 F & AGGGTGAAGCTCAGCTTG \\
ALA2b & ATGGTTAAAAAATCACTGCG \\
ALA3b & TTGCGCATACAGCGGCACAC \\
P2BC & CGCTGATTTAACAGATTCGG \\
PIAC & GGCGATCCGCGTGATACCAC \\
P2Dc & CAGCGCTTTTGCCGTCTAAG \\
MF-2 F & TGACGATGACCGCTTTCT \\
MF-2R & CGTCGACTACAGCTITAA \\
\hline
\end{tabular}


Table 2 PCR fragment sizes with defined primer combinations as calculated based on the chromosomal insertion*

\begin{tabular}{lll}
\hline Forward Primer & Reverse Primer & $\begin{array}{l}\text { Resulting PCR-Fragment } \\
\text { length in base pairs (bp) }\end{array}$ \\
\hline ALA2b & ALA3b & 88 \\
ALA2b & P2Bc & 290 \\
ALA2b & P2Dc & 941 \\
ALA2b & MP4b-F & 1516 \\
MP-3 F & DOW2.55 & 1156 \\
MP-3 F & DOW2.5 & 1718 \\
MP-3 F & ALA3b & 2078 \\
MP-3 F & P2Bc & 2280 \\
MP-3 F & P2Dc & 2931 \\
MP-3 F & MP4b-F & 3506 \\
P1AC & P2Dc & 410 \\
P1AC & MP4b-F & 985 \\
MF-2 F & MF-2R & 3435 \\
\hline
\end{tabular}

*When the insertion is not present at the examined locus, no specific PCR fragments should be able to be generated, except for the last primer pair, which should then give a PCR product of 459 base pairs (bp)

element was detected on the chromosome. The inverted repeats of th element were found by comparing the sequence to those of Partridge [21] and Fukui et al. (accession number AB701572.1). Primers were designed (Tables 1 and 2) to study the regions flanking CTX-M-15.

\section{Ethical approval}

The study was approved by the ethics committee of the medical faculty of the Justus-Liebig-University of Giessen (AZ: 95/11). All samples were taken as part of standard care procedures.

\section{Results}

ESBL alleles, susceptibility and genetic relatedness

PFGE displayed 3 clusters (B1-B3) using a similarity coefficient (SAB) of 0.8 (Fig. 1). Cluster B3 with 19 (83\%) isolates formed the largest group; within this cluster there were 10 isolates which had an identical PFGE pattern (SAB 1.0, sub-cluster A3). Cluster B3 isolates were assigned to sequence type 101 and cluster B2 to the recently reported ST628, while a representative isolate from cluster B1 was assigned to ST16.

All isolates were resistant to cefotaxime, ceftazidime, ceftriaxone, gentamicin, ciprofloxacin, trimethoprim /sulphamethaxazole and cefepime but sensitive to imipenem, meropenem and tigecycline. CTX-M genes were found in 19/23 of K. pneumoniae isolates. CTX-M-15 was the most common allele found in $16 / 23$ of isolates (Table 3). Isolate no. 71 had the same PGFE pattern as sub clone A3 isolates and phenotypically confirmed for ESBL production by disk approximation method. Even though the original strain was resistant to cefepime, on subculture it became sensitive

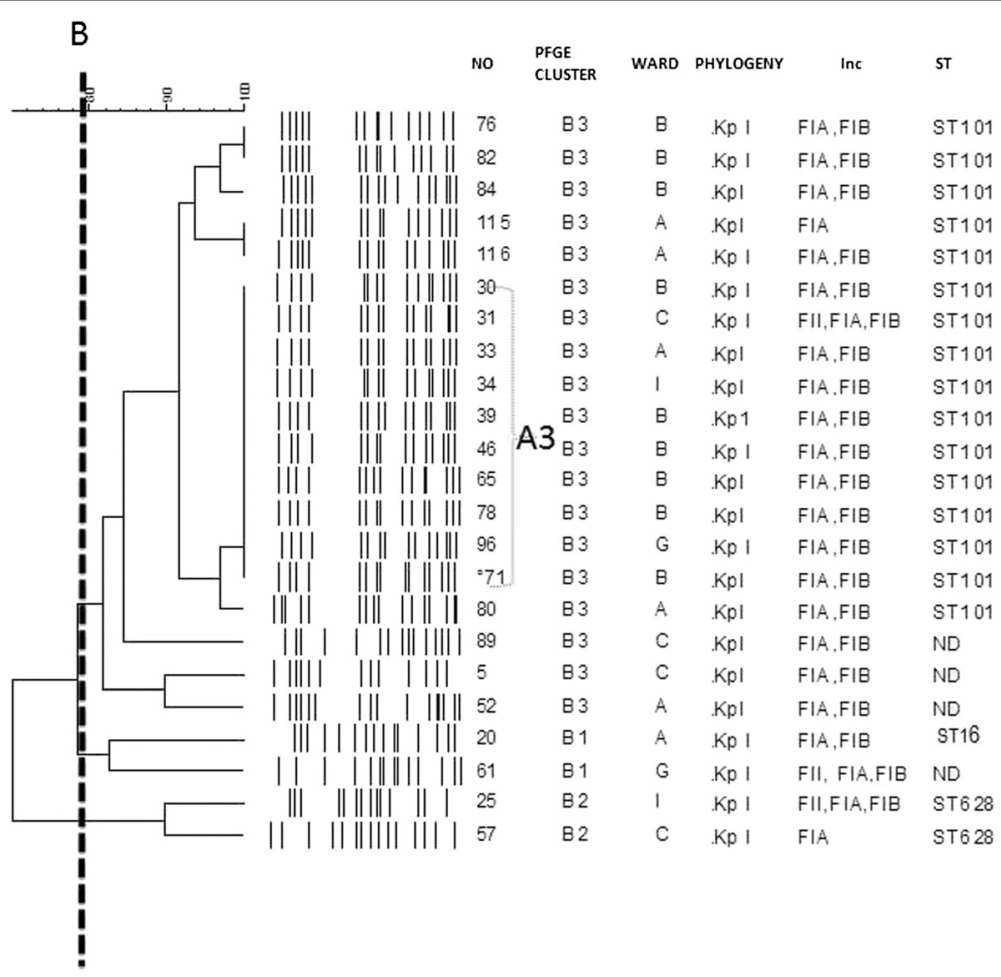

Fig. 1 Dendogram (UPGMA, DICE) showing the similarity for the 23 K. pneumoniae ESBL positive strains. Line B indicates $80 \%$ similarity. Note the clone A3, Phylogenetic group, wards A-I, PFGE clusters, isolate numbers and sequence type (ST) 
Table 3 Characteristics of the 23 K. pneumoniae isolates

\begin{tabular}{|c|c|c|c|c|c|}
\hline Isolate & Ward & PFGE cluster/group & ESBL type & Antibiotic resistance other than $\beta$-Lactams & Incompatibility group \\
\hline 20 & A & B1 & CTX-M-3, TEM-1 & GM, TET, SXT, CIP & FIA, FIB \\
\hline 61 & G & B1 & TEM-104 & GM, TET, SXT, CIP & FII, FIA, FIB \\
\hline 25 & । & B2 & TEM-54 & GM, TET, SXT, CIP & FIl, FIA, FIB \\
\hline 57 & C & B2 & CTX-M-15, TEM-1 & GM, TET & FIA \\
\hline 76 & B & B3 & CTX-M-15, TEM-1 & GM, TET, SXT, CIP & FIA, FIB \\
\hline 82 & B & B3 & CTX-M-15, TEM-1 & GM, TET, SXT, CIP & $\mathrm{FIA}, \mathrm{FIB}$ \\
\hline 84 & B & B3 & CTX-M-15, TEM-1 & GM, TET, SXT, CIP & FIA, FIB \\
\hline 115 & A & B3 & CTX-M-15, TEM-1 & GM, TET, SXT, CIP & FIA \\
\hline 116 & A & B3 & CTX-M-15, TEM-1 & GM, TET, SXT, CIP & FIA, FIB \\
\hline 30 & B & B3 & CTX-M-15, TEM-1 & GM, TET, SXT, CIP & $\mathrm{FIA}, \mathrm{FIB}$ \\
\hline 33 & A & B3 & CTX-M-15, TEM-1 & GM, TET, SXT, CIP & $\mathrm{FIA}, \mathrm{FIB}$ \\
\hline 34 & । & B3 & CTX-M-15, TEM-1 & GM, TET, SXT, CIP & $\mathrm{FIA}, \mathrm{FIB}$ \\
\hline 39 & B & B3 & CTX-M-15, TEM-1 & GM, TET, SXT, CIP & $\mathrm{FIA}, \mathrm{FIB}$ \\
\hline 31 & C & B3 & CTX-M-15, TEM-1 & GM, TET, SXT, CIP & $\mathrm{FIA}, \mathrm{FIB}$ \\
\hline 46 & B & B3 & CTX-M-15, TEM-1 & GM, TET, SXT, CIP & $\mathrm{FIA}, \mathrm{FIB}$ \\
\hline 65 & B & B3 & CTX-M-15, TEM-1 & GM, TET, SXT, CIP & $\mathrm{FIA}, \mathrm{FIB}$ \\
\hline${ }^{*} 71$ & B & B3 & TEM-1 & GM, TET, SXT, CIP & $\mathrm{FIA}, \mathrm{FIB}$ \\
\hline 78 & B & B3 & CTX-M-15, TEM-1 & GM, TET, SXT, CIP & $\mathrm{FIA}, \mathrm{FIB}$ \\
\hline 96 & G & B3 & CTX-M-15, TEM-1 & GM, TET, SXT, CIP & $\mathrm{FIA}, \mathrm{FIB}$ \\
\hline 80 & A & B3 & CTX-M-15, TEM-1 & GM, TET, SXT, CIP & $\mathrm{FIA}, \mathrm{FIB}$ \\
\hline 89 & C & B3 & CTX-M-3, TEM-1 & GM, TET, SXT, CIP & $\mathrm{FIA}, \mathrm{FIB}$ \\
\hline 5 & C & B3 & TEM-104 & GM, TET, SXT, CIP & ND \\
\hline 52 & A & B3 & CTX-M-3, TEM-1 & $\mathrm{GM}, \mathrm{SXT}, \mathrm{CIP}$ & FIA, FIB \\
\hline
\end{tabular}

*This isolate was positive for ESBL phenotypically but no ESBL gene was found

GM: Gentamicin, TET: Tetracycline, CIP: Ciprofloxacin, SXT: Sulphamethaxazole/trimethoprim, ND: not determined

with a MIC of $0.125 \mu \mathrm{g} / \mathrm{ml}$. The *71 isolate was found to lack the CTX-M15 gene.

\section{Plasmid analysis and chromosomal location}

Plasmid analysis revealed that all isolates harboured multiple plasmids of various sizes ranging from less than $48.5 \mathrm{~kb}$ to $436.5 \mathrm{~kb}$. Despite this fact, the CTX-M-15 resistance gene was not transferable from PFGE B3 sub cluster A3 isolates $(n=10)$ by conjugation or transformation in multiple attempts made. Hybridization using a CTX-M-15 DIG labelled probe indicated a chromosomal location in five isolates of PFGE type B3 subclone A3. Several colonies selected on a lysogeny broth plate containing $2 \mathrm{mg} / \mathrm{L}$ cefotaxime with a positive CTX-M PCR and phenotypically confirmed for ESBL production, were investigated for the insertion locus of the CTX-M-15 allele. In all cases a complete CTX-M-15/ISEcp1-element of 2,971 bp with flanking direct repeats (TCAAC) was found (Fig. $2 \mathrm{a} / \mathrm{b}$ ). Comparison of the contig sequence obtained by whole-genome sequencing of a representative isolate (no.39/URO33115) of the PFGEsub cluster A3, which harbours the CTX-M-15/ISEcp1-element, with a reference strain sequence ( $K$. pneumoniae 342 , accession number CP000964.1) revealed that the element is inserted between the coding sequences (CDS) KPK_0275 (hypothetical protein) and KPK_0276 (putative membrane protein, Fig. $2 \mathrm{a} / \mathrm{b}$ ), indicating integration of the transposition unit harbouring the ISEcp 1 insertion sequence and the CTX-M15 gene into the chromosome of the outbreak strain. The resulting contig sequence was deposited in the GenBank nucleotide database under accession number HG780615.

The other 9 strains within the sub-cluster A3 (ST101) were all tested positive for the same insertion locus using a specifically developed, sequence-based PCR-assay underlining their clonal identity (Table 2).

\section{Discussion}

The study documents the presence of $K$. pneumoniae clones carrying a chromosomally located CTX-M-15 in a large University Hospital in Germany. The high prevalence of CTX-M-15 is consistent with many other studies worldwide $[4,6]$ but unlike those studies in a subset of isolates, the CTX-M-15 gene was not transferable by conjugation or transformation and DNA hybridization 


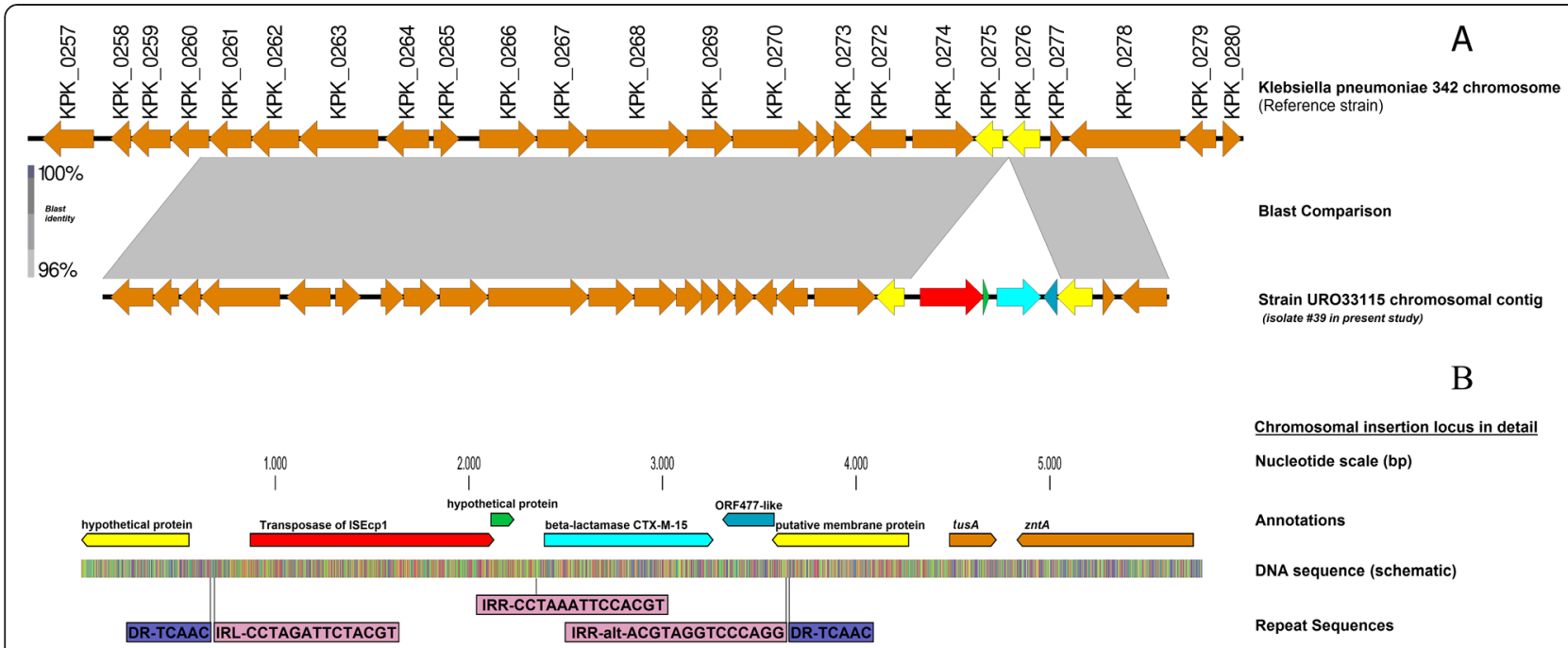

Fig. 2 Schematic diagram showing the chromosomal insertion site and the surrounding environment. a overview and chromosomal context compared to the reference genome of K. pneumoniae 342, accession number CP000964.1 b detailed view of the chromosomal insertion locus of

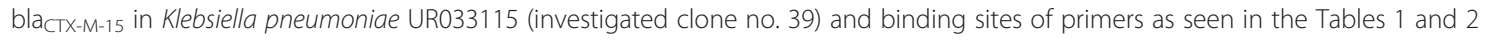

studies indicated a chromosomal localization for the gene. This was confirmed by whole genome sequencing and PCR mapping studies. Descriptions of chromosomal locations of ESBL genes in clinical isolates are extremely rare, with only a single report of chromosomally located CTX-M-15 at an undetermined location for a K. pneumoniae ST1 isolate and unlike the outbreak isolates reported here which were typed as ST101 [8]. ST101 belongs to the clonal complex 11; this clonal complex of K. pneumoniae has been associated with different ESBL alleles including CTX-M-15, OXA-48 and more recently $\mathrm{KPC}[22,23]$. It is now globally disseminated and is associated with healthcare-associated infections in North America, Europe and Asia [23-25]. It is worth noting that in most of the studies describing the epidemiology of $b l a_{\text {CTX-M-15, the location of the gene is not always }}$ stated, thus a chromosomal location may be a more common phenomenon than previously suspected.

In the present study the complete ISEcp $1 / \mathrm{CTX}-\mathrm{M}-15$ transposition unit with inverted repeats was found to be located on the chromosome, resulting in the duplication of a 5 bp repeat sequence (TCAAC). The organization of this transposition unit was similar as in conjugative IncF plasmids $[12,26]$ where the CTX-M-15 gene was also found $49 \mathrm{bp}$ downstream of ISEcp1. As described in reference plasmids the right inverted repeat was located in ORF477 (378 bp; GenBank accession no. HQ157357), downstream of the CTX-M-15 gene. This organization has been observed in $98 \%$ of IncF plasmids carrying CTX-M-15 and also in the chromosome of Kluyvera ascorbata carrying CTX-M-3 [27]. Chromosomally encoded $\beta$-lactamases from some Kluyvera species have been proposed as a possible source of CTX-M enzymes. The finding from this study suggests that other Enterobacteriaceae might also act as intermediate hosts and as a possible source of CTX-M genes.

\section{Conclusions}

For the first time we describe the site of integration of a complete CTX-M-15/ISEcp 1 element into the chromosome of a K. pneumoniae ST101 strain. This insertion site is distinct and differs markedly from a recently published sequence of a $\mathrm{K}$. pneumoniae ST11 isolate where CTX-M15 alleles were present both on a plasmid as well as a chromosomal location (CP006659.1, K. pneumoniae ATCC BAA-2146) [28]. The site of insertion also differs from that reported for the ST131 E. coli JJ1886 strain [29]. Our studies also reveal the presence of several large IncF plasmids in $K$. pneumoniae which have hitherto been poorly characterized, warranting additional studies. Using data derived in this study we have devised a specific PCRbased assay to examine for the chromosomal insertion of CTX-M-15 in ST101 K. pneumoniae isolates.

\section{Availability of supporting data}

The data set supporting the results of this article is available in GenBank under the accession number HG780615.

\section{Competing interests}

The authors declare that they have no competing interests.

\section{Authors' contributions}

SEM, Cl, ED and TC designed the study, SEM, Cl, MF and LF performed the experiments, SEM, Cl, TC analyzed the data, TH, Cl, MF, LF analyzed sequences, SEM, Cl, MF, LF and TC wrote the manuscript which was corrected and approved by all the other co-authors. 


\section{Acknowledgements}

The authors would like to acknowledge the technical support provided by the members of the Institute of Medical Microbiology Giessen. We thank Claudia Neumann, Isabell Trur, Kirsten Bommersheim, Christina Gerstmann and Alexandra Amend for their excellent technical assistance. This work was supported by a researcher startup grant of the Faculty of Medicine of the Justus-Liebig-University Giessen to $\mathrm{Cl}$ as well as by grants from the Bundesministerium fuer Bildung und Forschung (BMBF, Germany) within the framework of the RESET research network (contract no. 01KI1013G, 01KI1313G) and the German Center for Infection research (DZIF/grant number 8000 701-3 [HZl] to TC and $\mathrm{Cl}$ and Tl06.001 to TC).

\section{Author details}

${ }^{1}$ Catholic University of Health and Allied Sciences, Weill Bugando School of Medicine Box, 1464 Mwanza, Tanzania. ${ }^{2}$ Institute of Medical Microbiology, Justus-Liebig University, , Schubertstrasse 81, 35392 Giessen, Germany. ${ }^{3}$ German Center for Infection Research (DZIF), Partner site Giessen-Marburg-Langen, Campus Giessen, Schubertstrasse 81, 35392 Giessen, Germany.

Received: 15 January 2015 Accepted: 1 June 2015

Published online: 17 June 2015

\section{References}

1. Peirano G, Sang JHK, Pitondo-Silva A, Laupland KB, Pitout JD. Molecular epidemiology of extended-spectrum- $\beta$-lactamase-producing Klebsiella pneumoniae over a 10 year period in Calgary, Canada. J Antimicrob Chemother. 2012;67:1114-20

2. Romero L, Lopez L, Rodríguez-Baño J, Ramón Hernández J, MartínezMartínez L, Pascual A. Long-term study of the frequency of Escherichia col and Klebsiella pneumoniae isolates producing extended-spectrum B-lactamases. Clin Microbiol Infect. 2005;11:625-31.

3. Mshana SE, Hain T, Domann E, Lyamuya EF, Chakraborty T, Imirzalioglu C. Predominance of Klebsiella pneumoniae ST14 carrying CTX-M-15 causing neonatal sepsis in Tanzania. BMC Infect Dis. 2013;13:466.

4. Barguigua A, El Otmani F, Talmi M, Bourjilat F, Haouzane F, Zerouali K, et al. Characterization of extended-spectrum $\beta$-lactamase-producing Escherichia coli and Klebsiella pneumoniae isolates from the community in Morocco. J Med Microbiol. 2011;60:1344-52.

5. Poirel L, Bonnin RA, Nordmann P. Genetic support and diversity of acquired extended-spectrum $\beta$-lactamases in Gram-negative rods. Infect Genet Evol. 2012;12:883-93.

6. Younes A, Hamouda A, Dave J, Amyes S. Prevalence of transferable blaCTXM-15 from hospital-and community-acquired Klebsiella pneumoniae isolates in Scotland. J Antimicrob Chemother. 2011;66:313-8.

7. Coelho A, Mirelis B, Alonso-Tarrés C, Larrosa MN, Miró E, Abad RC, et al. Detection of three stable genetic clones of CTX-M-15-producing Klebsiella pneumoniae in the Barcelona metropolitan area, Spain. J Antimicrob Chemother. 2009;64:862-4.

8. Coelho A, González-López JJ, Miró E, Alonso-Tarrés C, Mirelis B, Larrosa MN, et al. Characterisation of the CTX-M-15-encoding gene in Klebsiella pneumoniae strains from the Barcelona metropolitan area: plasmid diversity and chromosomal integration. Int J Antimicrob Agents. 2010;36:73-8.

9. Fabre L, Delauné A, Espié E, Nygard K, Pardos M, Polomack L, et al. Chromosomal integration of the extended-spectrum $\beta$-lactamase gene blaCTX-M-15 in Salmonella enterica serotype Concord isolates from internationally adopted children. Antimicrob Agents Chemother. 2009;53:1808-16.

10. Song W, Kim J, Bae IK, Jeong SH, Seo YH, Shin JH, et al. Chromosome-encoded AmpC and CTX-M extended-spectrum $\beta$-lactamases in clinical isolates of Proteus mirabilis from Korea. Antimicrob Agents Chemother. 2011;55:1414-9.

11. Mshana SE, Imirzalioglu C, Hossain H, Hain T, Domann E, Chakraborty T. Conjugative IncFI plasmids carrying CTX-M-15 among Escherichia coli ESBL producing isolates at a University hospital in Germany. BMC Infect Dis. 2009;9:97.

12. Baraniak A, Fiett J, Hryniewicz W, Nordmann P, Gniadkowski M. Ceftazidimehydrolysing CTX-M-15 extended-spectrum $\beta$-lactamase (ESBL) in Poland. J Antimicrob Chemother. 2002;50:393-6.

13. Hunter PR, Fraser C. Application of a numerical index of discriminatory power to a comparison of four physiochemical typing methods for Candida albicans. J Clin Microbiol. 1989:27:2156-60

14. Hunter PR, Gaston MA. Numerical index of the discriminatory ability of typing systems: an application of Simpson's index of diversity. J Clin Microbiol. 1988;26:2465-6.
15. Brisse S, Van Himbergen T, Kusters K, Verhoef J. Development of a rapid identification method for Klebsiella pneumoniae phylogenetic groups and analysis of 420 clinical isolates. Clin Microbiol Infect. 2004;10:942-5.

16. Carattoli A, Miriagou V, Bertini A, Loli A, Colinon C, Villa L, et al. Replicon typing of plasmids encoding resistance to newer $\beta$-lactams. Emerg Infect Dis. 2006;12:1145.

17. Diancourt L, Passet V, Verhoef J, Grimont PA, Brisse S. Multilocus sequence typing of Klebsiella pneumoniae nosocomial isolates. J Clin Microbiol. 2005;43:4178-82

18. Schmitt J, Jacobs E, Schmidt H. Molecular characterization of extended-spectrum beta-lactamases in Enterobacteriaceae from patients of two hospitals in Saxony, Germany. J Med Microbiol. 2007;56:241-9.

19. Barton BM, Harding GP, Zuccarelli AJ. A general method for detecting and sizing large plasmids. Anal Biochem. 1995;226:235-40.

20. Chevreux B, Pfisterer T, Drescher B, Driesel AJ, Müller WE, Wetter T, et al. Using the miraEST assembler for reliable and automated mRNA transcript assembly and SNP detection in sequenced ESTs. Genome Res. 2004; 14:1147-59.

21. Partridge SR. Analysis of antibiotic resistance regions in Gram-negative bacteria. FEMS Microbiol Rev. 2011;35:820-55.

22. Potron A, Poirel L, Rondinaud E, Nordmann P. Intercontinental spread of OXA-48 beta-lactamase-producing Enterobacteriaceae over a 11-year period, 2001 to 2011. Euro Surveill. 2013;18:1-13.

23. Seki LM, Pereira PS, de Souza Mda P, Conceição MS, Marques EA, Porto CO, et al. Molecular epidemiology of KPC-2-producing Klebsiella pneumoniae isolates in Brazil: the predominance of sequence type 437. Diagn Microbiol Infect Dis. 2011;70:274-7.

24. Leavitt A, Carmeli Y, Chmelnitsky I, Goren MG, Ofek I, Navon-Venezia S. Molecular epidemiology, sequence types, and plasmid analyses of KPCproducing Klebsiella pneumoniae strains in Israel. Antimicrob Agents Chemother. 2010;54:3002-6.

25. Li B, Yi Y, Wang $Q$, Woo PC, Tan L, Jing $H$, et al. Analysis of drug resistance determinants in Klebsiella pneumoniae isolates from a tertiary-care hospital in Beijing, China. PloS One. 2012;7(7):e42280.

26. Boyd DA, Tyler S, Christianson S, McGeer A, Muller MP, Willey BM, et al. Complete nucleotide sequence of a 92-kilobase plasmid harboring the CTX-M-15 extendedspectrum beta-lactamase involved in an outbreak in long-term-care facilities in Toronto, Canada. Antimicrob Agents Chemother. 2004;48:3758-64.

27. Rodríguez MM, Power P, Radice M, Vay C, Famiglietti A, Galleni M, et al. Chromosome-encoded CTX-M-3 from Kluyvera ascorbata: a possible origin of plasmid-borne CTX-M-1-derived cefotaximases. Antimicrob Agents Chemother. 2004;48:4895-7.

28. Hudson CM, Bent ZW, Meagher RJ, Williams KP. Resistance Determinants and Mobile Genetic Elements of an NDM-1-Encoding Klebsiella pneumoniae Strain. PLoS One. 2014;9:e99209.

29. Andersen PS, Stegger M, Aziz M, Contente-Cuomo T, Gibbons HS, Keim P, et al. Complete genome sequence of the epidemic and highly virulent CTX-M-15-producing H30-Rx subclone of Escherichia coli ST131. Genome Announc. 2013;1:e00988-00913.

\section{Submit your next manuscript to BioMed Central and take full advantage of:}

- Convenient online submission

- Thorough peer review

- No space constraints or color figure charges

- Immediate publication on acceptance

- Inclusion in PubMed, CAS, Scopus and Google Scholar

- Research which is freely available for redistribution 